\title{
Cycle space constructions for exhaustions of flag domains
}

\author{
Alan HuckleberRy And Joseph A. Wolf
}

\begin{abstract}
A real semisimple group has only finitely many orbits on every flag manifold of its complexification. To each of these orbits there is a naturally associated space of algebraic cycles, and that cycle space is known to be a Stein manifold. In the past, properties of the cycle space have been proved by transforming functions or cohomology from, e.g., an open orbit in the flag manifold to its cycle space. Here the opposite is done: given an irreducible representation of a maximal compact subgroup of the real semisimple group, a canonical strictly plurisubharmonic exhaustion of the cycle space is constructed. This is then transformed to a (continuous) $q$-pseudoconvex exhaustion of the associated open orbit, where $q$ is the complex dimension of the cycles under consideration.
\end{abstract}

Mathematics Subject Classification (2010): 32M05 (primary); 32F10, 32M10, 22E46 (secondary).

\section{Introduction}

In the study of complex flag manifolds, flag domains and their cycle spaces, a key point is the fact that the cycle space $\mathcal{M}_{D}$ of a flag domain $D$ is a Stein manifold. That fact has a long history; see [4]. The earliest approach ([9], [11]) relied on construction of a strictly plurisubharmonic exhaustion function on $\mathcal{M}_{D}$, starting with a $q$-convex exhaustion function on $D$, where $q$ is the dimension of a particular maximal compact subvariety of $D$ (we use the normalization that 0 -convex means Stein). Construction of that exhaustion function on $D[8]$ required that $D$ be measurable [10]. In that case the exhaustion on $D$ was transferred to $\mathcal{M}_{D}$, using a special case of a method due to Barlet [2]. Here we do the opposite: we use the incidence method of [4] to construct a canonical plurisubharmonic exhaustion function on $\mathcal{M}_{D}$ and use it in turn to construct a canonical $q$-convex exhaustion function on $D$. This promises to have strong consequences for cohomology vanishing theorems and the construction of admissible representations of real reductive Lie groups.

Research partially supported by the DFG Sonderforschungsbereich "Symmetry and Universality in Mesoscopic Physics" and by NSF Grant DMS 99-88643.

Received October 28, 2008; accepted in revised form September 23, 2009. 
Our construction produces a continuous exhaustion of the flag domain $D$. It is $q$-pseudoconvex in the strong sense that it is optimally locally approximated by a smooth $q$-convex function. (See Section 5 for details.) Assuming that the AndreottiGrauert theory of pseudoconvexity and cohomology vanishing [1] remains valid for such exhaustions there will be direct applications to the geometric construction and analysis of admissible representations of real reductive Lie groups.

In this context we want to draw the reader's attention to the construction of Krötz and Stanton $([6,7])$ for plurisubharmonic exhaustion functions on $\mathcal{M}_{D}$ associated to spherical representations and their positive definite spherical functions.

Now we establish the basic notation of this paper. Let $G$ be a connected, simply connected complex semisimple Lie group, $\mathfrak{g}$ its Lie algebra, $\mathfrak{g}_{0}$ a real form of $\mathfrak{g}$, and $G_{0}$ the corresponding real form of $G$. Fix a parabolic subgroup $Q \subset G$ and let $Z$ denote the complex flag manifold $G / Q$. Then $G_{0}$ has only finitely many orbits on Z, in particular has open orbits [10]. By flag domain we mean an open orbit $D=G_{0}\left(z_{0}\right)$.

Fix a maximal compact subgroup $K_{0} \subset G_{0}$, let $K \subset G$ denote its complexification, and let $\mathfrak{k}_{0}$ and $\mathfrak{k}$ denote their respective Lie algebras. There is a unique $K_{0}$-orbit (call it $C_{0}$ ) in the flag domain $D$ that is a complex submanifold of $D$. Replacing $K_{0}$ by a $G_{0}$-conjugate if necessary we may assume that this base cycle $C_{0}=K_{0}\left(z_{0}\right)$, and then $C_{0}=K\left(z_{0}\right)$. The cycle space $\mathcal{M}_{D}$ is the topological component of $C_{0}$ in $\left\{g C_{0} \mid g \in G\right.$ and $\left.g C_{0} \subset D\right\}$.

Let $\theta$ denote the Cartan involution of $G_{0}$ with fixed point set $K_{0}$, and decompose $\mathfrak{g}_{0}=\mathfrak{k}_{0}+\mathfrak{s}_{0}$ into $( \pm 1)$-eigenspaces of $d \theta$. Then $\mathfrak{g}_{u}:=\mathfrak{k}_{0}+\sqrt{-1} \mathfrak{s}_{0}$ is a real subalgebra, in fact a real form, of $\mathfrak{g}$. The corresponding analytic subgroup $G_{u} \subset G$ is a compact real form of $G$.

We view the base cycle $C_{0}$ as a point in the complex space $\mathcal{C}_{q}(Z)$ of $q$ dimensional cycles in the projective algebraic variety $Z$. There the group $G$ acts algebraically as a group of holomorphic transformations, and consequently the orbit $\mathcal{M}_{Z}:=\left\{g C_{0} \mid g \in G\right\}$ is a complex $G$-homogeneous space. Since $D$ is open in $Z$, the subspace $\mathcal{M}_{Z} \cap \mathcal{C}_{q}(D)$ (cycles that are contained in $D$ ) is open in $\mathcal{M}_{Z}$, and $\mathcal{M}_{D}$ is its connected component containing $C_{0}$. In other words, $\mathcal{M}_{D}$ is the complex submanifold $\left\{g C_{0} \mid g \in G\right.$ and $\left.g C_{0} \subset D\right\}$ of $\mathcal{C}_{q}(D)$ as well as an open submanifold of $\mathcal{M}_{Z}$. In fact $\mathcal{M}_{D}$ is closed in $\mathcal{C}_{q}(D)$ [5]. As mentioned above, it is a Stein manifold.

The flag domain $D$ and its cycle space $\mathcal{M}_{D}$ fit into a holomorphic double fibration as follows. The incidence space $\mathfrak{X}_{D}:=\left\{(z, C) \in D \times \mathcal{M}_{D} \mid z \in C\right\}$ is a complex submanifold of $D \times \mathcal{M}_{D}$ and the projections are holomorphic fibrations:

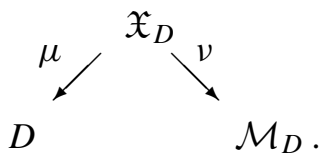

Note that $\mu$ is a holomorphic submersion and $v$ is a proper holomorphic map which is a locally trivial bundle. 


\section{Incidence geometry and Schubert fibrations}

Here we summarize several methods and results from our previous work which will be of use in the present note. These were systematically presented in [4]. The setting, as just described, is that of an open $G_{0}$-orbit $D$ in a complex flag manifold $Z=G / Q$. The maximal compact subgroup $K_{0}$ and the base cycle $C_{0}$ are constructed as above, and $q$ denotes the complex dimension of $C_{0}$.

Given an Iwasawa decomposition $G_{0}=K_{0} A_{0} N_{0}$, we write $A$ and $N$ for the complexifications of $A_{0}$ and $N_{0}$, and we consider Borel subgroups $B$ of $G$ that contain the solvable subgroup $A N$. Those are the Iwasawa Borel subgroups. Any two Iwasawa decompositions $G_{0}=K_{0} A_{0} N_{0}$ and $G_{0}=K_{0} A_{0}^{\prime} N_{0}^{\prime}$ are $K_{0}$-conjugate, and it follows that any two Iwasawa-Borel subgroups (for the same choice of $K_{0}$ ) are $K_{0}$-conjugate.

If $B \subset G$ is any Borel subgroup and $z \in Z$, then as usual we refer to the closure $S:=\mathrm{c} \ell(B(z))$ as the $S$ chubert variety associated to $B$ and $z$. The orbit $\mathcal{O}_{S}:=B(z)$, which is Zariski open in $S$, is the associated Schubert cell. The incidence variety $H_{S} \subset \mathcal{M}_{Z}$ consists of those cycles $g C_{0}, g \in G$, which have nonempty intersection with the boundary $B_{S}:=S \backslash \mathcal{O}_{S}$ of the Schubert cell in the Schubert variety. Below we will consider special points $z \in C_{0}$ where, when $B$ an Iwasawa-Borel subgroup relative to a decomposition $G_{0}=K_{0} A_{0} N_{0}$, the orbit $\Sigma:=A_{0} N_{0}(z)$ is exactly the connected component of $S \cap D$ that contains $z$. In that setting we refer to $\Sigma$ as a Schubert slice.

Since $C_{0}$ defines a nonzero class in $H_{2 q}(Z, \mathbb{Z})$, it has nonempty intersection with at least one $q$-codimensional $B$-Schubert variety $S$. Under the additional assumption that $B \supset A N$, such Schubert varieties have the following properties (see [4, Chapter II.7]):

- The intersection $C_{0} \cap S$ consists of finitely many points $z_{1}, \ldots, z_{m}$.

- These points are contained in the intersection $D \cap \mathcal{O}_{S}$ of $D$ with the Schubert cell (open $B$-orbit in $S$ ).

- For $1 \leqq j \leqq m$ the connected component $\Sigma_{j}$ of $S \cap D=\mathcal{O}_{S} \cap D$ which contains $z_{j}$ is the Schubert slice $A_{0} N_{0}\left(z_{j}\right)$.

- Every cycle $C \in \mathcal{M}_{D}$ intersects each Schubert slice $\Sigma_{j}$ in exactly one point.

- If $\Sigma$ is a Schubert slice then the incidence map $\pi_{\Sigma}: \mathcal{M}_{D} \rightarrow \Sigma$, which associates to $C$ its point of intersection with $\Sigma$, is an $A_{0} N_{0}$-equivariant holomorphic map that is a $C^{\infty}$-fiber bundle.

- The incidence variety $\mathcal{H}_{S}$ is a ( $B$-invariant) complex algebraic hypersurface in $\mathcal{M}_{Z} \backslash \mathcal{M}_{D}$.

If $C \in \mathcal{M}_{D}$ and $C \cap \Sigma=\{z\}$ then both the $\mu$-fiber $\mu^{-1}(z)$ of the double fibration and the $\pi_{\Sigma}$-fiber through $C$ defined just above can be identified with the set of all cycles which contain $z$.

Starting with $S$ and $H_{S}$ as above, we consider the union of all hypersurfaces $k\left(H_{S}\right)$ as $k$ runs over the compact group $K_{0}$. It is a basic fact that every boundary point of $\mathcal{M}_{D}$ in $\mathcal{M}_{Z}$ is contained in some such $k\left(H_{S}\right)$. This is formulated in [4] 
as follows. The union of the $k\left(H_{S}\right)$ as $k$ runs over $K_{0}$ is a closed subset of $\mathcal{M}_{Z}$ and the connected component of its complement containing $\mathcal{M}_{D}$ is a $G_{0}$-invariant, Kobayashi hyperbolic, Stein domain $\mathcal{E}\left(H_{S}\right)$ (see [4, Chapter 11]). The desired result is [4, Theorem 11.3.1]; it states that $\mathcal{M}_{D}$ is the maximal $G_{0}$-invariant, Kobayashi hyperbolic, Stein domain in $\mathcal{M}_{Z}$ which contains the base cycle $C_{0}$. This implies $\mathcal{M}_{D}=\mathcal{E}\left(H_{S}\right)$.

For our purposes it is useful to formulate the above fact as follows. For every cycle $C$ in the boundary of $\mathcal{M}_{D}$ in $\mathcal{M}_{Z}$ there exist a Schubert slice $\Sigma$ and an element $k \in K_{0}$ so that $C$ is both in the boundary of $k(\Sigma)$ and the boundary $\mathcal{B}_{k(S)}$ of $\mathcal{O}_{k(S)}$ in $k(S)$.

Of course it is possible for a sequence $\left\{C_{n}\right\}$ to be divergent in $\mathcal{M}_{D}$ without converging to a boundary point $C \in \mathcal{M}_{Z}$. But in this case, after taking a subsequence, we may regard it as converging to a point $C$ in the "wonderful compactification" of $\mathcal{M}_{Z}$. Even in that case one observes that $C$ is in $\mathcal{B}_{k(S)}$, i.e. is in the boundary of some $k(\Sigma)([5])$.

\section{Exhaustions of Schubert cells}

As above $Z$ denotes the complex flag manifold $G / Q$ where $G$ is a connected, simply connected, complex semisimple group and $Q$ is a parabolic subgroup. Thus every holomorphic line bundle $\mathbb{L} \rightarrow Z$ is canonically a $G$-bundle. We now assume that $\mathbb{L}$ is positive. Then the action of $G$ on the space $\Gamma(Z, \mathbb{L})$ of holomorphic sections is an irreducible finite dimensional representation.

Since $\mathbb{L}$ is a line bundle, the isotropy subgroups of $G_{u}$ are irreducible on its fibers, so $\mathbb{L} \rightarrow Z$ has a unique (up to multiplication by a positive real constant) $G_{u}$-invariant hermitian metric. We write $\|\cdot\|$ for the associated norm function on sections of $\mathbb{L} \rightarrow Z$.

Given a section $s \in \Gamma(Z, \mathbb{L})$ the function $\eta_{s}:=-\log \|s\|^{2}$ is a strictly plurisubharmonic exhaustion of the complement of the zero set $\{s=0\}$ in $Z$.

Now let $B$ be a Borel subgroup of $G$ and $S=\mathcal{O}_{S} \cup \dot{\cup} B_{S}$ a $B$-Schubert variety. Let $\iota_{S}: S \rightarrow Z$ be the canonical embedding and $V_{S}:=\iota_{S}^{*}(\Gamma(Z, \mathbb{L}))$ the space of restricted (to $S$ ) sections. Since $V_{S}$ is the representation space for a rational representation of $B$, we have $B$-eigenvectors $s \in V_{s}$. If $U$ is the unipotent radical of $B$, then any two such eigenvectors are $U$-invariant and their ratio is a $U$-invariant meromorphic function on $S$. Since $U$ has an open orbit in $S$, that function is constant, so the two eigenvectors are linearly dependent.

Proposition 3.1. Let $\mathbb{L} \rightarrow Z$ be a positive holomorphic line bundle, $B \subset G a$ Borel subgroup, and $S \subset Z$ a $B$-Schubert variety. Then any nonzero B-eigenvector $s \in V_{S}$ defines a strictly plurisubharmonic exhaustion $r_{S}:=\left.\eta_{s}\right|_{\mathcal{O}_{S}}$ of the open $B$-orbit in $S$.

Proof. It remains only to show that $s$ vanishes exactly on $B_{S}$. For this see [4, Corollary 7.4.9]. 
It should be underlined that having fixed $\mathbb{L}$ and the maximal compact subgroup $G_{u}$, the exhaustion function $r_{S}$ is unique.

\section{The minimax construction}

Here we first construct continuous plurisubharmonic exhaustions $r_{\mathcal{M}_{D}}$ of $\mathcal{M}_{D}$ by taking the supremum of lifted exhaustions $r_{S} \circ \pi_{\Sigma}$ defined by natural families of Schubert fibrations $\pi_{\Sigma}: \mathcal{M}_{D} \rightarrow \Sigma \hookrightarrow S$. We then lift $r_{\mathcal{M}_{D}}$ to the universal family $\mathfrak{X}_{D}$ and push this function down to a continuous $q$-convex exhaustion $r_{D}$ of $D$ by taking the infimum over the fibers of $\mu: \mathfrak{X}_{D} \rightarrow D$.

\subsection{Lifting Schubert exhaustions}

As in Section 2 let $B$ be a Borel subgroup of $G$ which contains an Iwasawa component $A N$, and let $S$ be a $B$-Schubert variety which is of complementary dimension to that of the base cycle $C_{0}$ and which has nonempty intersection with $C_{0}$. Recall that $\operatorname{dim}\left(C_{0}\right):=q$ and that $D$ is $n$-dimensional so that $\operatorname{dim}(S)=n-q$. Furthermore, the intersection $S \cap D$ is contained in the open $B$-orbit $\mathcal{O}_{S}$ in $S$ and the components $\Sigma$ of this intersection are orbits $A_{0} N_{0}(z)$ of the intersection points $z \in S \cap C_{0}$.

We fix a Schubert slice $\Sigma$. If $C \in \mathcal{M}_{D}$ then $C \cap \Sigma$ consists of a single point. Thus $\Sigma$ defines a fibration $\pi_{\Sigma}: \mathcal{M}_{D} \rightarrow \Sigma$ by $C \mapsto C \cap \Sigma$. The relevant family of Schubert varieties is defined by allowing $B$ to vary under the condition that it contains $A N$. In fact this is just the closed $G_{0}$-orbit in $G / B$, in other words the orbit on which $K_{0}$ acts transitively. Hence the family of Schubert varieties determined by some initial choice $S_{0}$ is just $\left\{k\left(S_{0}\right) \mid k \in K_{0}\right\}$. Thus we are interested in the family $\left\{k(\Sigma) \mid k \in K_{0}\right\}$ of Schubert slices.

If the exhaustion $r_{S}$ of $S$ is defined as above by the section $s$, then the exhaustion $r_{k(S)}$ of $k(S)$ is defined by $k(s)$. Hence

$$
r_{k(S)} \circ \pi_{k(\Sigma)}(C)=r_{S} \circ \pi_{\Sigma}(k(C)) .
$$

Provisionally define $r_{\mathcal{M}_{D}}$ to be the supremum of the plurisubharmonic functions $r_{k(S)} \circ \pi_{k(\Sigma)}$. Since $\Sigma$ is just one connected component of $S \cap D$, the final definition of $r_{\mathcal{M}_{D}}$ is given by maximizing over all such components.

Proposition 4.1. The function $r_{\mathcal{M}_{D}}: \mathcal{M}_{D} \rightarrow \mathbb{R}$ is a $K_{0}$-invariant continuous plurisubharmonic exhaustion.

Proof. It is a general fact that the supremum of a family of plurisubharmonic functions is plurisubharmonic. It follows from (4.1) that the provisionally defined function arises by maximizing a fixed continuous function over a compact group action. Hence that function is continuous and, as the maximum of finitely many such functions, it then follows that $r_{\mathcal{M}_{D}}$ is continuous. Since we have taken the supremum over a $K_{0}$-invariant family of functions, $r_{\mathcal{M}_{D}}$ is also $K_{0}$-invariant. 
Finally, every boundary point of $\mathcal{M}_{D}$ in the affine homogeneous space $G / K$ is contained in some $K_{0}$-translate $k\left(H_{0}\right)$ of the incidence hypersurface $H_{0}$ defined by $B_{S_{0}}$ ([4]; see Section 2 above). This implies that if $\left\{C_{m}\right\}$ is a sequence in $\mathcal{M}_{D}$ which converges to such a boundary point, then $r_{\mathcal{M}_{D}}\left(C_{m}\right) \rightarrow \infty$. If the sequence $C_{m}$ diverges in $G / K$, then we may assume that it converges to a point in the wonderful compactification of $G / K$ which is also in such a translate $k\left(H_{0}\right)$ ( [5]). Thus $r_{\mathcal{M}_{D}}\left(C_{m}\right) \rightarrow \infty$ for any sequence $\left\{C_{m}\right\}$ which diverges in $\mathcal{M}_{D}$ and therefore $r_{\mathcal{M}_{D}}$ is an exhaustion.

\subsection{Transfer to the flag domain}

The map $v: \mathfrak{X}_{D} \rightarrow \mathcal{M}_{D}$ is proper; in fact, it is a trivial fiber bundle with fiber $C_{0}$. Thus the lift $r_{\mathfrak{X}_{D}}:=r_{\mathcal{M}_{D}} \circ v$ is a proper exhaustion. Transferring to the flag domain, we define $r_{D}: D \rightarrow \mathbb{R}$ by

$$
r_{D}(y):=\inf \left\{r_{\mathfrak{X}_{D}}(x) \mid x \in \mu^{-1}(y)\right\} .
$$

The following follows from the fact that $r_{\mathfrak{X}_{D}}$ is proper and $\mu: \mathfrak{X}_{D} \rightarrow D$ is an open map.

Lemma 4.2. The function $r_{D}: D \rightarrow \mathbb{R}$ is a continuous $K_{0}$-invariant exhaustion.

Proof. Since $r_{\mathfrak{x}_{D}}$ is $K_{0}$-invariant and $\mu: \mathfrak{X}_{D} \rightarrow D$ is $K_{0}$-equivariant, it is immediate that $r_{D}$ is $K_{0}$-invariant.

To prove that $r_{D}$ is continuous, we let $\left\{y_{m}\right\} \rightarrow y$ in $D$. Since $r_{\mathfrak{X}_{D}}$ is a continuous exhaustion, $r_{D}\left(y_{m}\right)=r_{\mathfrak{x}_{D}}\left(x_{m}\right)$ for some $x_{m} \in \mu^{-1}\left(y_{m}\right)$ and we may assume that $\left\{x_{m}\right\} \rightarrow x$. This is due to the fact that if $\left\{x_{m}\right\}$ is unbounded in $\mathfrak{X}_{D}$, then $v\left(x_{m}\right)$ is unbounded in $\mathcal{M}_{D}$ and as a result

$$
r_{D}\left(y_{m}\right)=r_{\mathfrak{X}_{D}}\left(x_{m}\right)=r_{\mathcal{M}_{D}} \circ v\left(x_{m}\right) \rightarrow \infty .
$$

On the other hand, $\varlimsup r_{D}\left(y_{m}\right)$ is bounded by $r_{D}(y)$. For similar reasons, if $r_{D}(y)$ were not $r_{\mathfrak{X}_{D}}(x)$, then there would exist $\tilde{x} \in \mu^{-1}(y)$ with $r_{D}(y)=r_{\mathfrak{X}_{D}}(\tilde{x})<$ $r_{\mathfrak{x}_{D}}(x)$. But $\mu$ is an open map and, contrary to assumption, we would be able to find $\tilde{x}_{m} \in \mu^{-1}\left(y_{m}\right)$ with $r_{\mathfrak{x}_{D}}\left(\tilde{x}_{m}\right)<r_{\mathfrak{X}_{D}}\left(x_{m}\right)$. Thus $r_{D}$ is continuous.

To see that $r_{D}$ is an exhaustion, let $\left\{y_{m}\right\}$ be a divergent sequence in $D$ and choose $\left\{x_{m}\right\}$ with $x_{m} \in \mu^{-1}\left(y_{m}\right)$ and $r_{D}\left(y_{m}\right)=r_{\mathfrak{X}_{D}}\left(x_{m}\right)$. It follows that $\left\{x_{m}\right\}$ is divergent as well, and since $r_{\mathfrak{X}_{D}}$ is an exhaustion, $r_{D}\left(y_{m}\right) \rightarrow \infty$.

\section{Pseudoconvexity}

Since $r_{D}$ is only known to be continuous, it doesn't make sense to discuss its Levi form at a point of the boundary of $D_{r}:=\left\{r_{D}<r\right\}$. In the case of continuous 
plurisubharmonic exhaustions this causes no difficulties. However, as is pointed out in [3], subtle difficulties arise in the $q$-convex case.

Let us say that a continuous exhaustion $h: D \rightarrow \mathbb{R}$ is $q$-pseudoconvex if every $p \in D$ has an open neighborhood $U$ equipped with a smooth function $\tilde{h}$ with $\tilde{h}(p)=h(p), \tilde{h} \leqq h \mid U$, and the Levi form $\mathcal{L}(\tilde{h})=\frac{i}{2} \partial \bar{\partial} \tilde{h}$ has at least $n-q$ positive eigenvalues at every point of $U$.

In particular the region $\{\tilde{h}<\tilde{h}(p)\}$ contains $D \cap U$ and has a common boundary point with it at $p$, and near $p$ it has smooth $q$-convex boundary. In this sense our definition of a continuous $q$-pseudoconvex function can be regarded as being natural. For the purposes of smoothing, so that for example the theorems of Andreotti and Grauert can be directly applied, it may be necessary to require a somewhat stronger property. It is quite possible that our exhaustion is indeed more regular than we have shown, but proving this in detail will require further study.

Theorem 5.1. The exhaustion $r_{D}: D \rightarrow \mathbb{R}$ is q-pseudoconvex.

Proof. Let $y \in\left\{r_{D}=r\right\}$. Let $x \in \mathfrak{X}_{D}$ be such that $r_{D}(y)=r_{\mathfrak{X}_{D}}(x)$ and recall that $x=(y, C)$ with $y \in C$. Now by definition $r_{\mathfrak{x}_{D}}(x)=r_{\mathcal{M}_{D}}(C)$, and $r_{\mathcal{M}_{D}}(C)$ can be computed as $r_{S} \circ \pi_{\Sigma}(C)$, for some Schubert variety $S$ in the defining family and some slice $\Sigma$. Since $r_{\mathcal{M}_{D}} \geqq r_{S} \circ \pi_{\Sigma}$, it follows that $\left\{r_{\mathcal{M}_{D}} \leqq r\right\} \subset\left\{r_{S} \circ \pi_{\Sigma} \leqq r\right\}$.

Now lift $r_{S} \circ \pi_{\Sigma}$ and $r_{D}$ to functions $h_{S}$ and $h_{D}$ on $\mathfrak{X}_{D}$. We consider these functions in a small neighborhood $W$ of $x=(y, C)$. Note that they agree on the $\mu$-fiber $\mathcal{F}:=\mu^{-1}(y)$. Furthermore, $h_{D} \geqq h_{S}$ on a neighborhood of $(y, C)$ and thus we may assume that this holds on $W$.

By shrinking $W$ if necessary, we may choose a closed complex submanifold $T$ of $W$ which is transversal to $\mathcal{F}$ and is mapped biholomorphically onto its open image $U$ in $D$. Define $r_{T}$ on $U$ by restricting $h_{S}$ to $T$ and using the identification $T=U$. Finally, choose $T$ in such a way that it contains a closed $(n-q)$ dimensional complex submanifold $\Delta$ through $x$ so that the restriction of $\pi_{\Sigma} \circ v$ to $\Delta$ is biholomorphic onto an open subset of $\Sigma$. These choices can be made, because $\mathcal{F}$ is both the $\mu$-fiber over $y$ and the $\pi_{\Sigma}$-fiber through $C$. By construction $\left\{r_{T} \leqq r\right\}$ contains $D_{r} \cap U$. Furthermore, its restriction to the $\mu$-image of $\Delta$ can be regarded as the restriction of $r_{S}$ to the $\pi_{\Sigma} \circ v$-image of $\Delta$. Since $r_{S}$ is strictly plurisubharmonic, it follows that $\mathcal{L}\left(r_{T}\right)$ has the desired number of positive eigenvalues.

\section{References}

[1] A. ANDREOTTI and H. GRAUERT, Théorèmes de finitude pour la cohomologie des espaces complexes, Bull. Soc. Math France 90 (1962), 193-259.

[2] D. Barlet, Convexité de l'espace des cycles, Bull. Soc. Math. France 106 (1978), 373-397.

[3] M. G. Eastwood and G. V. Souria, Cohomologically complete and pseudoconvex domains, Comment. Math. Helv. 55 (1980), 413-426.

[4] G. Fels, A. T. Huckleberry and J. A. Wolf, "Cycle Spaces of Flag Domains: A Complex Geometric Viewpoint”, Progress in Mathematics, Vol. 245, Birkhäuser, Boston, 2006. 
[5] J. Hong and A. T. Huckleberry, On closures of cycle spaces of flag domains, Manuscripta Math. 121 (2006), 317-327.

[6] B. KRÖTZ and R. STANTON, Holomorphic extension of representations, I. Automorphic functions, Ann. of Math. 159 (2004), 641-724.

[7] B. KRÖTZ and R. STANTON, Holomorphic extension of representations, II. Geometry and harmonic analysis, Geom. Funct. Anal. 15 (2005), 190-245.

[8] W. SCHMID and J. A. WoLF, A vanishing theorem for open orbits on complex flag manifolds, Proc. Amer. Math. Soc. 92 (1984), 461-464.

[9] R. O. Wells, JR., and J. A. WOLF, Poincaré series and automorphic cohomology on flag domains, Ann. of Math. 105 (1977), 397-448.

[10] J. A. WoLf, The action of a real semisimple Lie group on a complex manifold, I. Orbit structure and holomorphic arc components, Bull. Amer. Math. Soc. 75 (1969), 1121-1237.

[11] J. A. WOLF, The Stein condition for cycle spaces of open orbits on complex flag manifolds, Ann. of Math. 136 (1992), 541-555.

[12] J. A. WOLF, Exhaustion functions and cohomology vanishing theorems for open orbits on complex flag manifolds, Math. Res. Lett. 2 (1995), 179-191.

Fakultät für Mathematik Ruhr-Universität Bochum D-44780 Bochum, Germany ahuck@cplx.rub.de

Department of Mathematics University of California Berkeley, California 94720-3840, U.S.A. jawolf@math.berkeley.edu 